\title{
Design and development of sEMG Prosthetics for recovering amputation of the human hand
}

\author{
Masood-ur-Rehman*, Ajab Khan Kasi, Jafar Khan Kasi, Muzamil \\ Bokhari and Muhammad Sohail \\ Department of Physics, University of Balochistan, Sariab Road Quetta-Pakistan \\ *Corresponding author's email: masoodphys@gmail.com \\ Citation
}

Masood-ur-Rehman, Ajab Khan Kasi, Jafar Khan Kasi, Muzamil Bokhari1 and Muhammad Sohail. Design and development of sEMG Prosthetics for recovering amputation of the human hand. Pure and Applied Biology. Vol. 8, Issue 3, pp1935-1942. http://dx.doi.org/10.19045/bspab.2019.80137

\begin{tabular}{llll}
\hline \hline Received: 15/05/2019 & Revised: 17/07/2019 & Accepted: 19/07/2019 & Online First: 23/07/2019 \\
\hline \hline
\end{tabular}

\section{Abstract}

The upper limb amputation is one of the biggest human health problems that have a great psychological, social and economic effect on the life of a person. The motorized prosthetics give a better solution for amputees. Unfortunately, in developing countries such as Pakistan, the poor amputees cannot afford expensive prosthetics which is mostly developed in European and American countries. This paper reports the low-cost solution of the prosthetic limb. A simple design of prosthetic hand is reported which is developed using 3D printing technology. This prosthetic hand has five degrees of freedom in which each finger can be actuated individually. The hand is actuated using surface electromyographic (sEMG) signals from working muscles located near amputation point. This hand has capability to sense and display the temperature of anybody in contact. The main components of this prosthetic hand are the sEMG sensor, Arduino Nano Microcontroller, five servo motors, and temperature sensor. There are 5 grasping modes which enables the prosthetic hand to give different motions using single EMG signals. This hand was tested successfully for all five modes and its performance was good in picking and dropping objects from one box to another.

Keywords: 3D printing; Amputation; EMG signal; Polylactic Acid; Upper limb prosthesis

\section{Introduction}

Amputation is a process in which a part of the human body is removed in the result of any problem. The major causes of amputation are congenital diseases, cancer, road accident, and terrorist attacks. According to the literature, there are approximately two million amputees in the United States [1]. In Pakistan the database of amputees is not maintained however according to some surveys, Trauma, with $80 \%$, is one of the major causes of amputation in Pakistan [2]. The upper limb amputation is one of the extreme human health problems that have a great psychological, social and economic effect on the life of a person. The amputation can be overcome by replacing the missing limb with an artificial limb, called prosthesis that can mimic the functionality of an original limb to some extent such as grasping of an object. Electromyography (EMG) is the widely used method to control the upper limb prosthesis.

The EMG signals are being in use in prosthetic hand since 1948 when Reinhold Reiter created $1^{\text {st }}$ myo electric hand. But due to bulky vacuum tube electronics setup, it didn't get commercial and clinical appreciation [3]. In 1960, a Russian 
scientist Koberinski made $1^{\text {st }}$ clinically acceptable transradial (below elbow) myoelectric prosthesis. It used transistor and battery that runs the relay to control the motion of motors [4]. In 1980, the myoelectric prostheses were in use in rehabilitation centers throughout the world, but their functions were restricted to one degree of freedom (DoF) and performed only opening and closing moments of fingers [5]. Though these state-of-the-art prostheses had facilitated the amputees, but it had some limitations like poor control, less functionality, maintenance, fabrication cost, weight and lack of sensory feedback [6]. So, a lot of work has been done to overcome these limitations and to improve the performance of prostheses that are useful in activities of daily life (ADL). The smart hand as reported by Cipriani et al., used 4 brushed D.C motors to actuate all five fingers with $16 \mathrm{DoF}$. It had 40 force, pressure and position sensors in fingers and embedded controller that automatically control the grasp and deliver the feedback to amputee [7]. Pisa /IIT SoftHand reported by Catalano et al., actuated 19 joints of prototype prosthetic hand by using a single actuator [8].

Lack of sensory feedback is one of the major reasons of rejection of the prosthetic hands as the prosthetic users desire to rely less on visual information. Number of researchers has developed different feedback methods that include vibrational feedback, electro tactile feedback, mechanotactile pressure, temperature feedback, audio feedback and augmented reality. Some researchers developed a hybrid model by combining two or more than two feedback techniques [9].

Commercially a lot of work has been done in this field as compared to the research field. But very limited of them have the capability to replicate various movements of the original hand. The top companies and approaches that are presently manufacturing the most advanced hand prostheses are (1) Touch Bionics i-limb Quantum; (2) Otto Bock Michelangelo; (3)
Steeper Bebionic v3; and (4) Vincent hand Evolution2. These hands have great performance in ADLs and have a better feedback mechanism, but the commercially available prosthetic hands are expensive and are not affordable for people in developing countries. In Pakistan, none of the company or research center is providing motorized prosthetic hands which are frustrating for upper limb amputees [10, $11]$.

In this paper, we have designed and developed motorized prosthetic hand according to the lifestyle, living standard and economical condition of amputees in Pakistan and sensing the temperature using the vibrational feedback system in the device. It is $3 \mathrm{D}$ printed and sEMG controlled prosthetic hand that has 5 actuators having 5 DoFs.

\section{Materials and methods}

The schematic diagram of proposed prosthetic hand system is depicted in (Figure 1). It comprises of three sEMG sensor electrodes, myoware muscle sensor (signal conditioner), Arduino Nano Microcontroller, five servo motors, fishing wires, and an negative temperature coefficient (NTC) thermistor analog temperature sensor.

Three sEMG electrodes sense the muscles signals, that is amplified, filtered and rectified with the help of myoware muscle sensor. That signal is then processed with the help of Arduino Nano that is used to drive five servo motors fitted in the palm of the hand. These five servo motors drive the five fingers of the prosthetic hand with the help of fishing wires. An NTC thermistor is used to sense the temperature. The detail of major parts is given below.

\section{EMG signal acquisition}

Three sEMG Sensor electrodes are used to detect the EMG signal which is placed on the forearm muscles. These electrodes adhere to the skin with the help of adhesive gel and do not fall but can easily be detached. The initial EMG signals are of very low voltage, so with the help of signal conditioner, these signals are amplified, 
filtered and rectified. The signal conditioner is integrated into MyoWare Muscle Sensor which was purchased from advancer technology. The signal conditioner used in this project is small in size, low weight, low cost and easy to operate. The MyoWare sensor gives $0-5 \mathrm{~V}$ analog which reflects the muscles actuation. The output of sensor is fed in Arduino Nano microcontroller where it proceeds to control servomotors.

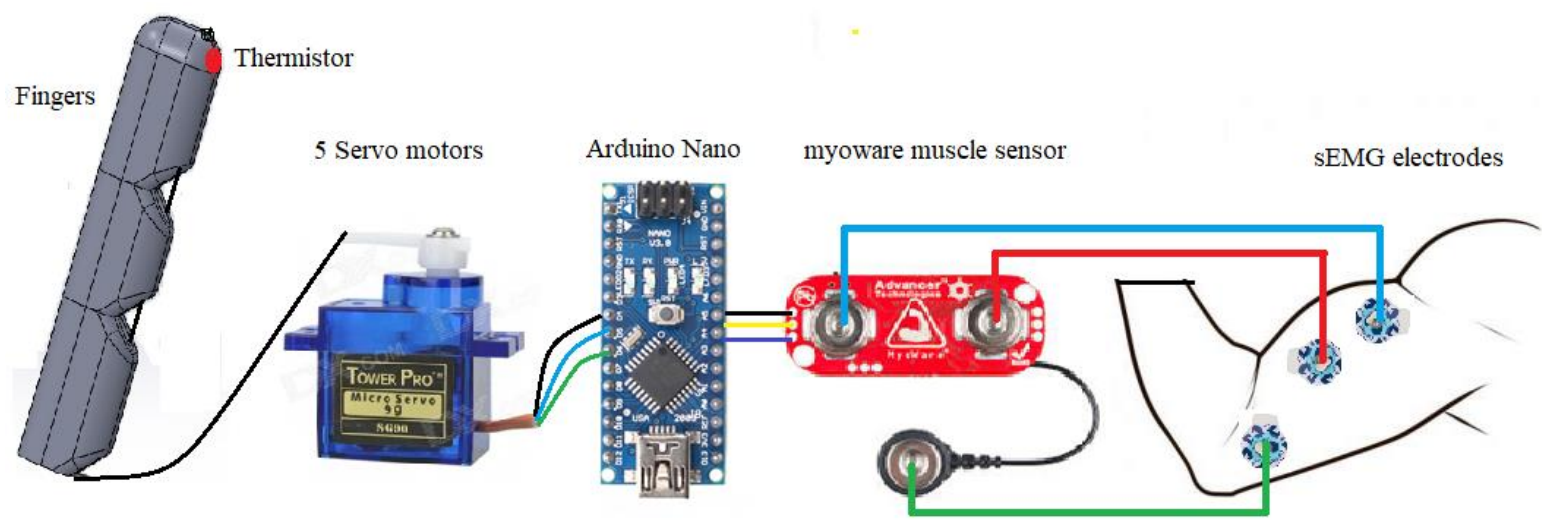

Figure 1. Schematic of prosthetic hand electronic parts

\section{Mechanical design}

The 3D design of prosthetic hand is shown in (Figure 2). The mechanical part of device is designed in solids work and printed using $3 \mathrm{D}$ printer. The $3 \mathrm{D}$ printing technology is widely used in making of upper limb prosthesis from more than 6 years [12]. The $3 \mathrm{D}$ structure of the prosthetic hand was designed in $\mathrm{CAD}$ format and then converted into STL which was further translated in G-
Code which was readable by $3 \mathrm{D}$ printer. The 3D design consists of 5 fingers and a palm. Each finger has three joints that can rotate up to 90 degrees. The palm of the hand is designed such that all servo motors can be fitted inside it. Each finger is connected with palm with the help of a rubber band. For actuation, these fingers are further connected with servo motors using fishing wire.

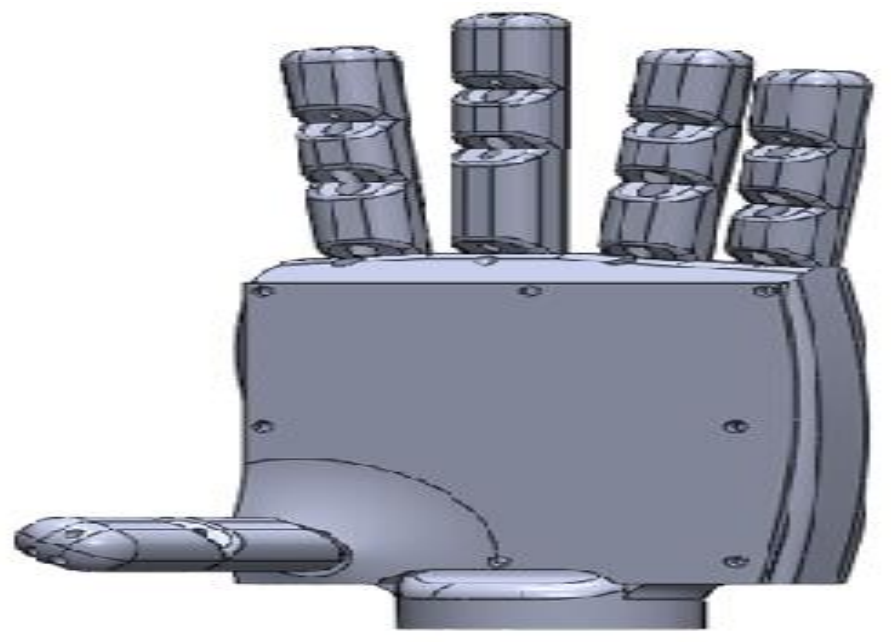

Figure 2. 3D design of prosthetic hand 
Fused Deposition Modelling technology (FDM) was used to print the 3D model of the prosthetic hand. In FDM the thermoplastic polymer in the form of filament is heated with the help of thermally controlled head, that ejects the molten material and place layer by layer on the moveable table [13]. For this purpose, JGAurora A5 3D printer is used. Polylactic Acid (PLA) is used as printing material. This material is used because it is lightweight and strong enough. All parts of hand are made and printed separately and then connected with each other. Fishing wire is used for the flexion of the finger while a rubber band is used for the extension of the finger. One end of fishing wire and the rubber band was tied with the fingertip, thread through each joint of the finger and the other end of fishing wires was tied with the rotating part of the servo motor. 5 servo motors were used to actuate all five fingers. When the motor rotates in one direction it stretches the fishing wire to produce flexion and as it is rotated in reverse direction the rubber band pulls back the finger to produce extension.

The heart of the project is Arduino Nano, which is used to control the whole system. It read the sensor value at its analog input and can change it into output with the help of PWM pins. It has 14 digital input-output pins which are used to run all five servo motors. A 6V, 3800mAh Ni MH battery has been used to power all the circuitry.

\section{Temperature sensing}

A feedback sensing network is used to scene the temperature of the environment. An NTC thermistor along with a 10k resistor is used as an analog temperature sensor for sensing the temperature. The Thermistor is used because it is small and can easily fit in the fingertip of the index finger as shown in (Figure 3). When this thermistor senses the temperature, it sends the data to the analog input of Arduino which convert it into Celsius temperature with the help of programming [14]. 5 LEDs are used to indicate the change in temperature.

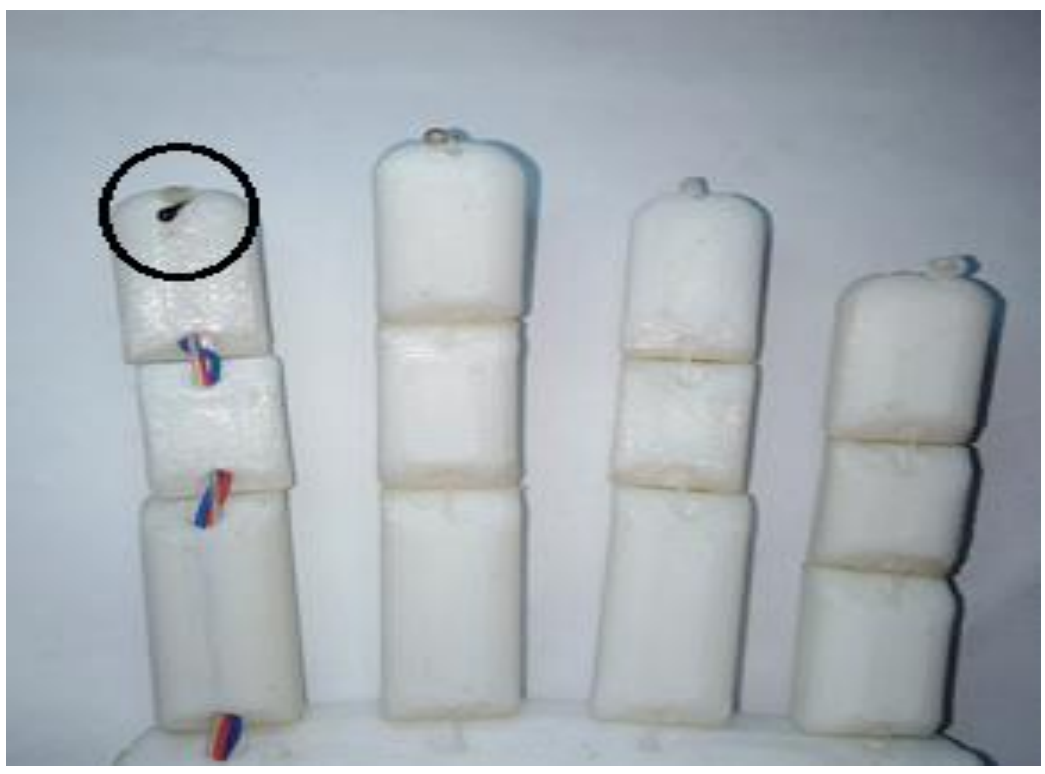

Figure 3. Thermistor in the index finger

\section{Result and discussion}

The completely assembled prosthetic hand shown in (Figure 4). It consists of a 3D printed hand, myoware muscle sensor, circuit board, and battery. The prosthetic hand uses myoelectric signals for actuation.
Three sEMG electrodes sense this signal from residual limb. The initial signal from EMG electrodes is in the form of very low voltage with different frequencies. So, it is amplified filtered and rectified with the help of myoware muscle sensor up to $5 \mathrm{~V}$. The 0 - 
5V analog DC signal from the myoware sensor is sent to Arduino Nano microcontroller. The Arduino is an opensource platform that can be easily programmed to perform various operations on data set and provide control to attached hardware with and has simple hardware system [13]. The Arduino Nano is programmed in Arduino integrated development environment (IDE) software. In this project, the Arduino was programmed according to the algorithm given in (figure 5).

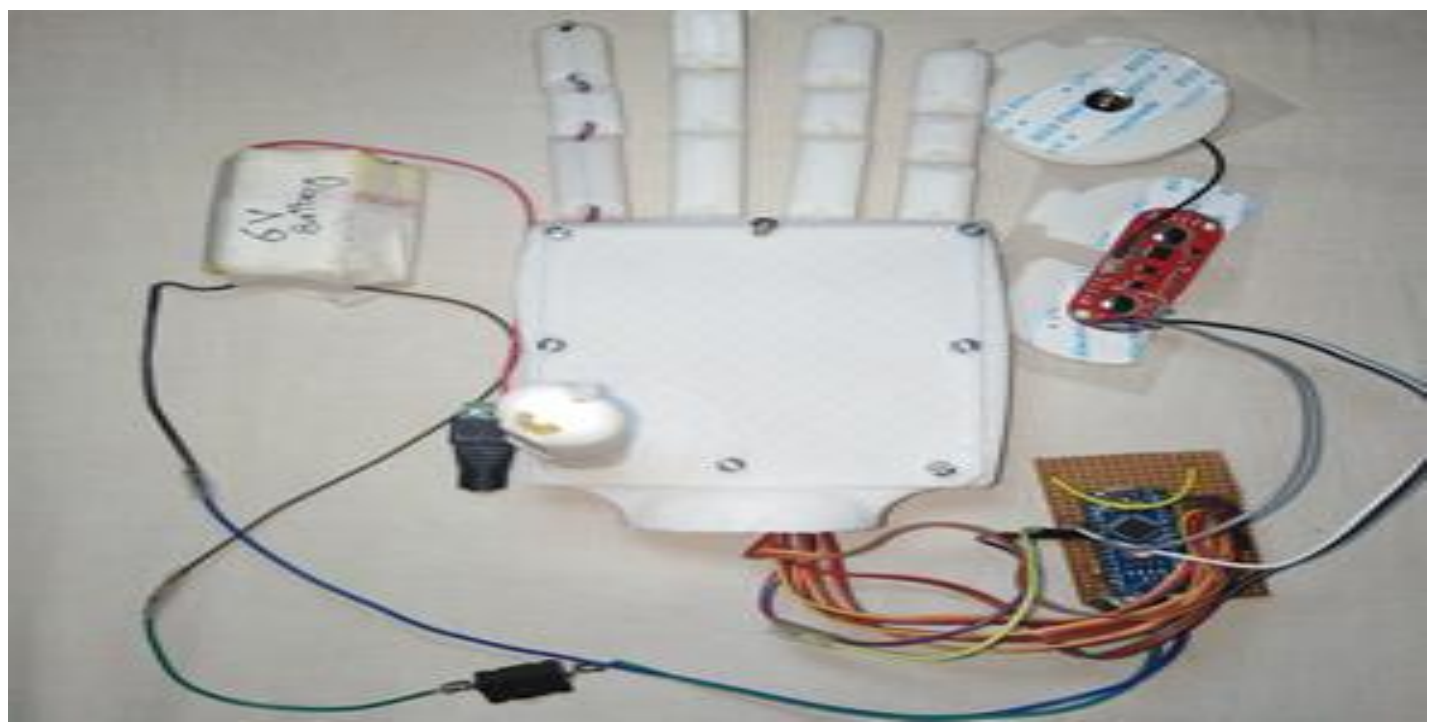

Figure 4. Completely assembled prosthetic hand, sensor, circuit board, and battery

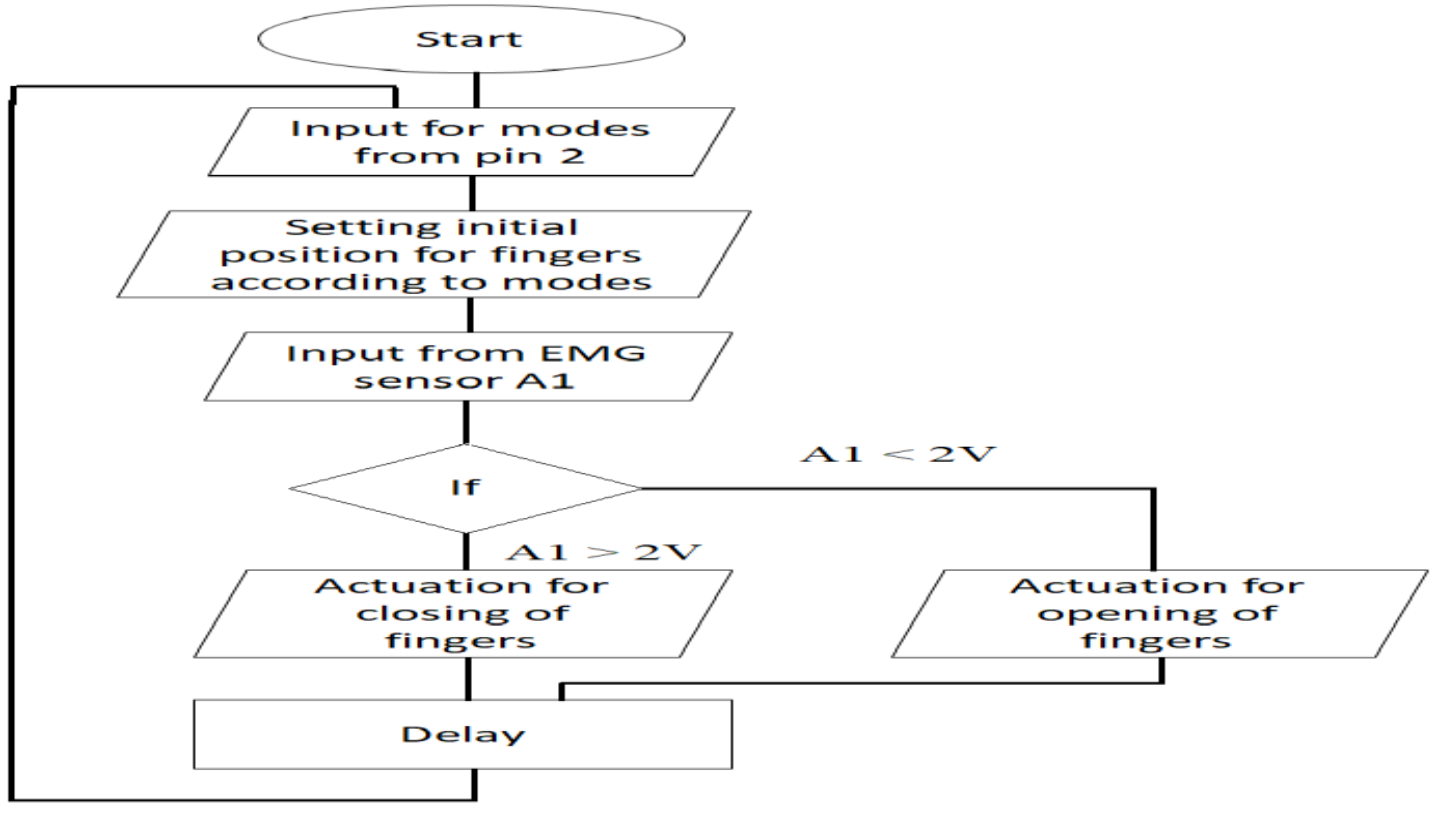

Figure 5. Algorithm for programming Arduino for hand actuation

Initially, the Arduino takes input from mode input: 2 which decides the mode of the prosthetic hand. There are 5 modes which enable the prosthetic hand to give different motions using a single EMG sensor. These are 1) griping mode, 2) thumb up mode 3) Ok sign mode, 4) victory sign mode and 5) Pin holding mode. When any mode is selected the microcontroller gives the signal to servo motors to fix initial position of the 
hand (fingers) in the decided mode. In next step, the controller takes signal from the EMG sensor according to which the required fingers are actuated. After some delay, the entire process is repeated to get new signals from EMG sensor. After receiving the signal from EMG sensor, the microcontroller decides whether fingers are actuated to get open or closed state. Five servo motors fitted inside the palm to actuate all five fingers. Flexion of the fingers or closing of hand is done with the help of fishing wires that are thread through the joints of fingers. One end of the fishing wires are tied at the tips of fingers while other ends are attached to the servo motors. When a signal is generated from myoware muscle sensor to the Arduino, then the Arduino sends a command to the servo motors to rotate 180 degrees, so it pulls the fishing wires. As a result, flexion is produced in the fingers and the hand is closed. When there is no signal generated then the Arduino sends a command to the servo motors to go back to its initial position. The extension in the fingers or opening of hand is produced with the help of rubber bands that are thread through back sides of joints of fingers and tied at the back sides of the hand. These rubber bands pull back the fingers and brings them to erect position. The movement of the hand in each mode is shown in (Figure 6).

The hand successfully performed all five modes. These modes were selected to allow an amputee to perform activities of daily life. This hand is then tested for grasping different objects such as (a) tennis ball, (b) mobile phone (c) energy drink can and (d) banana as shown in (Figure 7).

An NTC thermistor in the fingertip is used to sense the temperature. It is small in size and can easily fit in the fingertip. The thermistor is attached with A2 of the Arduino microcontroller. Steinhart-hart equation is used in programming to get the precise temperature of the thermistor which is given below;

$T=\frac{1}{A+B \ln (R)+C[\ln (R)]^{3}}$

Where $\mathrm{T}$ is temperature, $\mathrm{A}, \mathrm{B}$ and $\mathrm{C}$ are coefficients. $\mathrm{R}$ is the resistance of the thermistor at $\mathrm{T}$ temperature $[14,15]$. The temperature of the sensor is displayed on vertical LED segments such that each segment represents a $10^{\circ} \mathrm{C}$ increase in temperature. The $1^{\text {st }}$ LED segment is turned on at $30{ }^{\circ} \mathrm{C}, 2^{\text {nd }}$ at $40{ }^{\circ} \mathrm{C}$ and so on.

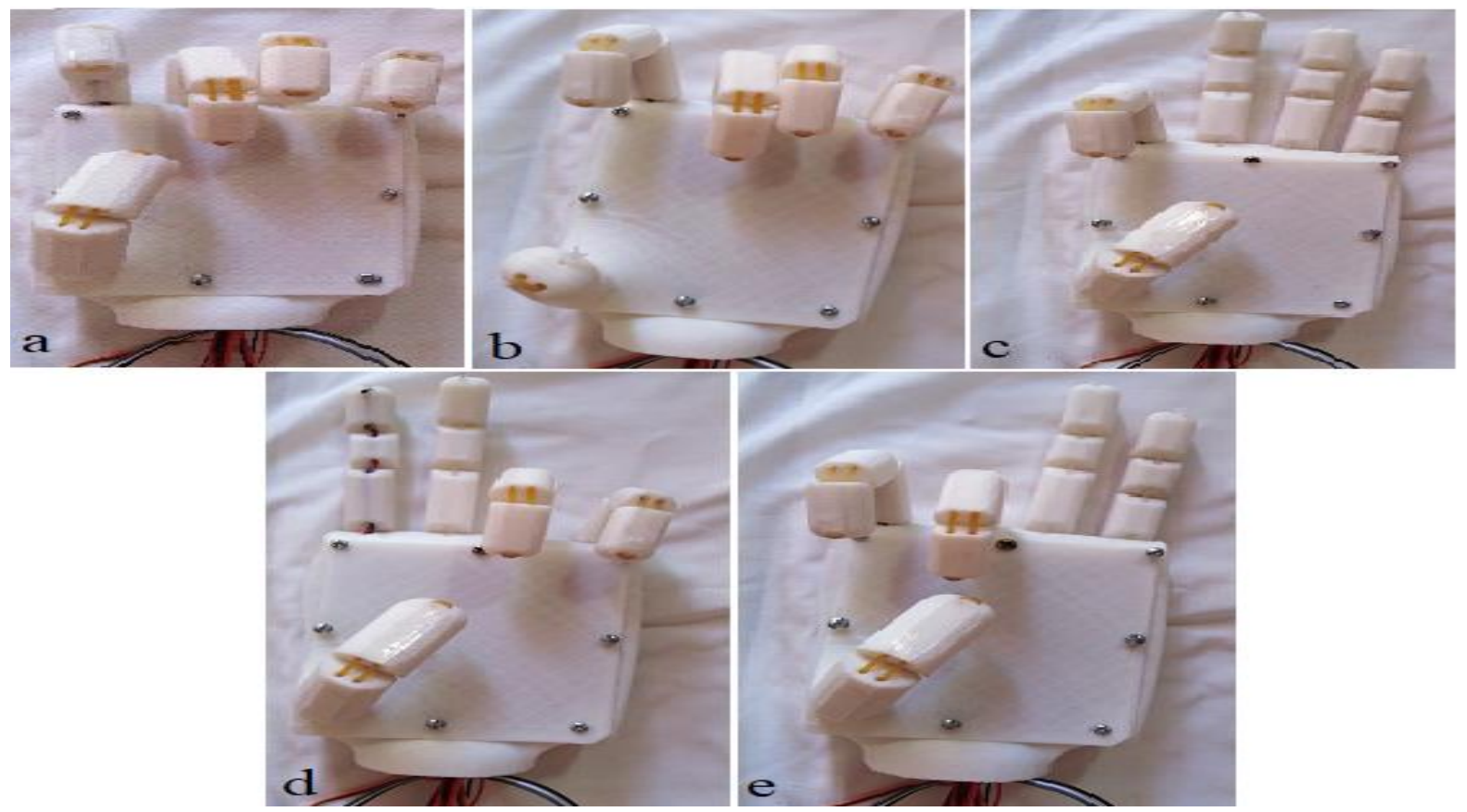

Figure.6 The prosthetic hand movement in 1) griping mode, 2) thumb up mode 3) Ok sign mode, 4) victory sign mode and 5) Pin holding mode 


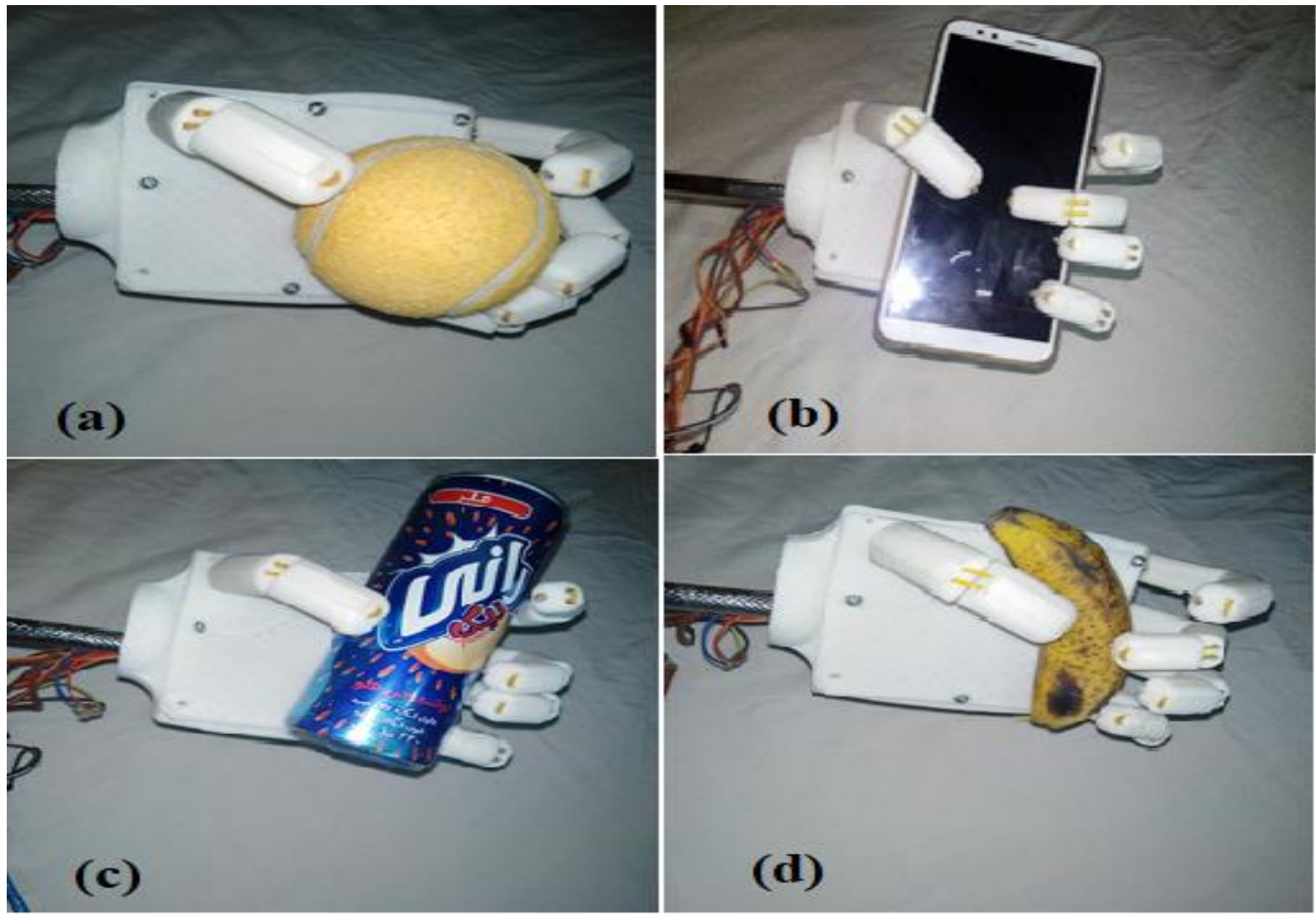

Figure 7. Hand test for griping different objects (a) tennis ball, (b) mobile phone (c) energy drink can and (d) banana

\section{Conclusion}

The main purpose of this research was to develop a low-cost prosthetic hand that is according to the lifestyle, living standard and economic conditions of amputees in developing countries like Pakistan. The implementation of simple design approach, control algorithm and hardware system made it possible to develop a low-cost prosthetic hand. The components for this prosthetic hand are of low cost and easily available in Pakistan. 3D printing technology has been adopted in making this prosthetic hand which is a versatile and easy approach. The hand has been tested for five different grasping modes by using myoware muscle sensor. The addition of temperature sensor includes the sensing capabilities of the prosthetic hand which is resembled the human hand. This hand will be a ray of hope for the poor amputees in developing countries like Pakistan and it will entirely change their social life.

\section{Authors' contributions}

Conceived and designed the experiments: M Rehman \& AK Kasi, Performed the experiments: M Rehman, Analyzed the data: AK Kasi \& M Rehman, Contributed materials/ analysis/ tools: JK Kasi, M Bokhari \& M Rehman, Wrote the paper: M Rehman, AK Kasi \& M Sohail.

\section{References}

1. Benatti S, Milosevic B, Farella E, Gruppioni E, \& Benini L (2017). A prosthetic hand body area controller based on efficient pattern recognition control strategies. Sensors 17(4): 869.

2. Shahzad A, Malik LA, Hussain H \& Soomro SK (2016). CAUSES OF AMPUTATION IN PAKISTANI POPULATION. Inter $J$ of Rehabilitation Sci (IJRS) 5(2): 54-57.

3. Geethanjali P (2016). Myoelectric control of prosthetic hands: state-ofthe-art review. Medical Devices (Auckland, NZ) 9: 247. 
4. Sherman ED (1964). A Russian bioelectric-controlled prosthesis: Report of a research team from the Rehabilitation Institute of Montreal. Canadian Medical Assoc J91(24): 1268.

5. Zuo KJ \& Olson JL (2014). The evolution of functional hand replacement: From iron prostheses to hand transplantation. Plastic Surgery 22(1): 44-51.

6. Biddiss EA \& Chau TT (2007). Upper limb prosthesis use and abandonment: a survey of the last 25 years. Prosthetics and Orthotics Inter 31(3): 236-257.

7. Cipriani C, Controzzi M \& Carrozza MC (2011).The SmartHand transradial prosthesis. $J$ of Neuroenginr and Rehabilitation 8(1): 29.

8. Catalano MG, Grioli G, Farnioli E, Serio A, Piazza C \& Bicchi A (2014). Adaptive synergies for the design and control of the Pisa/IIT SoftHand. The Inter J of Robotics Res 33(5): 768-782.

9. Stephens-Fripp B, Alici G \& Mutlu R (2018). A review of non-invasive sensory feedback methods for transradial prosthetic hands. IEEE Access 6: 6878-6899.
10. Belter JT, Segil JL \& SM B (2013). Mechanical design and performance specifications of anthropomorphic prosthetic hands: a review. $J$ of Rehabilitation Res and Devel 50(5): 599.

11. Atzori M \& Müller H (2015). Control capabilities of myoelectric robotic prostheses by hand amputees: a scientific research and market overview. Frontiers in Sys Neurosci 9: 162.

12. Ten Kate J, Smit G \& Breedveld $P$ (2017). 3D-printed upper limb prostheses: a review. Disability and Rehabilitation: Assistive Technol 12(3): 300-314.

13. Bonini $\mathrm{N}$, Iyer $\mathrm{N}$, Kim $\mathrm{D}$, Mathison $\mathrm{K}$ \& Wellons L (2014). Robotic Hand in Motion Using Arduino-Controlled Servos. New Jersey Governor's School of Engineering and Technology.

14. Recktenwald G (2013). Temperature Measurement with a Thermistor and an Arduino. Pdx. Edu.

15. Kinchin J (2018). Using an Arduino and cheap thermistor to make a simple temperature sensor. Physics Edu 53(6): 063008. 